\title{
RELACIÓN ENTRE EL NIVEL DE SENSIBILIDAD PERIFÉRICA, OBESIDAD Y LOS PARÁMETROS DE LA MARCHA Y DE EQUILIBRIO EN ADULTOS MAYORES CON DIABETES TIPO 2
}

\author{
THE RELATIONSHIP BETWEEN LEVEL OF PERIPHERAL \\ SENSITIVITY, OBESITY AND GAIT AND BALANCE PARAMETERS IN \\ OLDER ADULTS WITH TYPE-2 DIABETES
}

\author{
Elizabeth Guzmán Ortiz \\ Berta Cecilia Salazar González ${ }^{* *}$
}

\begin{abstract}
RESUMEN
Objetivo: Conocer la relación entre la sensibilidad de los pies, índice de masa corporal (IMC), y los parámetros de la marcha y de equilibrio en adultos mayores entre 65 y 75 años de edad. Material y método: Estudio descriptivo, correlacional y transversal, con una muestra de 119 adultos mayores con diagnóstico de diabetes tipo 2 (DT2). Se aplicaron las siguientes mediciones: el sistema GAITRite ${ }^{\circledR}$ Electronic Walkway para los parámetros de la marcha, la Batería Corta de Desempeño Físico para equilibrio y monofilamento de Semmes-Weinstein, North Coast Medical de 10 gramos para la sensibilidad y mediciones antropométricas para IMC. Se realizó un análisis estadístico con pruebas no paramétricas. Resultados: Se encontraron diferencias significativas en la velocidad, longitud del paso, de la zancada y el equilibrio entre los adultos mayores que tenían sensibilidad disminuida y normal. Conclusión: Una menor sensibilidad de los pies y mayor IMC se relacionan con mayor alteración de los parámetros de la marcha y el equilibrio en adultos mayores de 65 a 75 años de edad con DT2.
\end{abstract}

Palabras clave: Adulto mayor, diabetes mellitus tipo 2, marcha, equilibrio postural.

\begin{abstract}
Objective: To determine the relationship between feet sensitivity, body mass index (BMI), and gait and balance parameters in older adults between 65 and 75 years of age. Material and methods: A descriptive, correlational and cross-sectional study with a sample of 119 older adults diagnosed with type 2 diabetes (DT2). The following measurements were applied: the GAITRite ${ }^{\circledR}$ Electronic Walkway system for gait parameters, the Short Physical Performance Battery for balance, the Semmes-Weinstein monofilament examination, the North Coast Medical test using 10 grams for foot sensitivity and anthropometric measurements for BMI. A statistical analysis with non-parametric tests was performed. Results: Significant differences were found in speed, step length, stride length and balance between older adults who had decreased and normal sensitivity. Conclusion: Less foot sensitivity and a higher BMI are associated with a greater impairment reflected by the walking and balance parameters in older adults aged 65 to 75 with DT2.
\end{abstract}

Key words: Elderly, diabetes mellitus type 2, gait, postural balance.

Fecha recepción: 02/06/15 Fecha aceptación: 23/11/16

*Enfermera. Lic. Estudiante de maestría en ciencias de Enfermería. Universidad Autónoma de Nuevo León, México. E-mail: eliza_8_9@hotmail.com

${ }^{* *}$ Enfermera. PhD. Docente. Universidad Autónoma de Nuevo León, México. E-mail: bceci195@hotmail.com 


\section{INTRODUCCIÓN}

A escala mundial, la proporción de personas mayores de 60 años de edad aumentó del 9\% en 1994 al 12\% en 2014, y se espera que alcance el $21 \%$ en 2050 (1). Así también las tendencias indican que para ese año el número de personas de edad avanzada que no puedan valerse por sí mismas se multiplicará por cuatro en los países en desarrollo (2).

En México los Adultos Mayores (AM) constituyen el 9,5\% de la población total, proporción que implica más de 10 millones de AM (3). De esa proporción más de una cuarta parte $2.690 .000(26,9 \%)$ presenta algún grado de discapacidad, al menos para realizar una actividad básica de la vida diaria y la principal limitación se refiere a problemas para caminar (18,4\%) (4). La limitación para caminar representa un problema social y de salud que implica disminución de la autonomía del adulto mayor entre otros problemas.

La limitación de movilidad se puede deber a cambios asociados al envejecimiento que incluyen reducción de la fuerza muscular, limitación del rango de movimiento de las articulaciones, alteración en los tiempos de reacción y del sistema sensorial $(5,6)$. Estos cambios aunados a alguna patología como la diabetes tipo 2 (DT2) pueden afectar con mayor severidad a los sistemas músculo-esquelético y nervioso, lo que a su vez provoca efectos negativos en el control del equilibrio y la marcha en los adultos mayores $(7,8)$ predisponiéndolos a caídas y fracturas.

La marcha se define como un proceso de locomoción en el cual el cuerpo humano, en posición erecta generalmente, se desplaza hacia delante, siendo su peso soportado alternativamente por ambos miembros inferiores (9). En la marcha se incluye el equilibrio, como componentes de la buena locomoción del adulto mayor.

El control de equilibrio se define como la capacidad de mantener el centro de masa del cuerpo dentro de la base de soporte. Para que se establezca el equilibrio se requiere una interacción de los sistemas músculo-esquelético y neuronal. Los componentes músculo-esqueléticos incluyen la amplitud de movimiento articular, flexibilidad de la columna y las propiedades musculares y los componentes neuronales incluyen procesos motores, procesos sensoriales/perceptuales (10). El deterioro de estos sistemas con el envejecimiento puede conducir al deterioro del equilibrio y caídas.

Existen diversos factores que afectan los parámetros de la marcha y el equilibrio, entre ellos la presencia de DT2, particularmente el desarrollo de neuropatía periférica (NP), obesidad y personas en edad avanzada (11). La NP afecta todos los nervios periféricos, incluyendo fibras del dolor, neuronas motoras, y el sistema nervioso autónomo. La NP implica disminución de la sensibilidad y pérdida de reflejos, debido a que las fibras nerviosas largas se ven afectadas en mayor proporción que las fibras nerviosas cortas, es decir que la conducción nerviosa disminuye en proporción a la longitud del nervio (12). El daño neuromuscular, consecutivo a la neuropatía, altera la biomecánica de las extremidades inferiores, lo que a su vez lleva a alteraciones de la marcha y equilibrio con el aumento del riesgo de caídas (13), particularmente la disminución de la velocidad se asocia a caídas y muerte prematura (14). Investigaciones muestran inconsistencias acerca de que las alteraciones de la marcha y el equilibrio son mayores en pacientes con neuropatía que en aquellos que no desarrollan esta complicación (13, 15-17).

Por otra parte, reportes de investigación señalan que la obesidad se asocia con una disminución de la longitud de la zancada, cadencia y velocidad al caminar, aumento en el tiempo total de la postura durante un ciclo de la marcha e inestabilidad postural (18-20). Lo anterior se puede explicar por el aumento de la carga mecánica y dinámica en los patrones de movimiento de la rodilla (21-23). 
La evidencia de los factores asociados a los parámetros de la marca en los adultos mayores con DT2, enfatiza la responsabilidad del personal de salud de la valoración de los niveles de sensibilidad de los pies y control del peso corporal en el adulto mayor con DT2, con la finalidad de ofrecer medidas de protección y prevención de riesgos de caídas. Se considera un área de oportunidad para que el personal de enfermería en el primer nivel de atención coordine sus acciones con el personal médico y cuidadores del AM con DT2, a fin de seguir la evolución del paciente. Difundir los factores asociados permite contribuir en los indicadores del dominio $11 \mathrm{de}$ seguridad y protección de los diagnósticos enfermeros (24).

Por tal razón, el objetivo del estudio fue conocer la relación entre la sensibilidad de los pies, índice de masa corporal (IMC), y los parámetros de la marcha y equilibrio en adultos mayores entre 65 y 75 años de edad.

\section{MATERIAL Y MÉTODO}

El diseño del estudio fue descriptivo, correlacional y transversal. Se incluyeron 119 adultos mayores de 65 a 75 años de edad con diagnóstico de DT2, que asistían a las casas club del adulto mayor del estado de Nuevo León. Se seleccionaron a aquellos con capacidad para caminar y estar de pie solos o con ayuda de algún dispositivo, así como para escuchar la voz del entrevistador. Se excluyeron a los adultos que expresaron dolor de rodillas al caminar y presentaron desorientación en espacio, tiempo o persona; este se evaluó a través de preguntas tales como nombre, edad, día y lugar donde se encontraba en ese momento.

El muestreo fue de tipo no probabilístico, por cuota. El tamaño de muestra se calculó mediante el paquete nQuery Advisor versión 4.0 , con una potencia del $90 \%$, nivel de sig- nificancia de .05 , tamaño de efecto mediano (25).

\section{Mediciones}

Los datos se registraron en una cédula de datos, compuesta por tres apartados, en el primero se registraban los datos personales del participante: edad, sexo, estado marital, años de escolaridad y remuneración económica. El segundo apartado comprendía los antecedentes patológicos expresados por el adulto mayor y los años de padecer alguna enfermedad. En el último apartado se registraban las mediciones clínicas (valoración de la marcha y equilibrio y sensibilidad de los pies y el peso corporal).

\section{Mediciones clínicas}

La marcha de los pacientes fue capturado en el sistema GAITRite ${ }^{\oplus}$ Electronic Walkway, que mide y registra los parámetros espaciales y temporales de la marcha. Consta de un tapete compuesto de sensores sensibles a la presión, tiene una longitud de $550 \mathrm{~cm}$ y un ancho de $90 \mathrm{~cm}$. Éste se conecta vía USB a un ordenador portátil equipado con un software, que registra los datos obtenidos de los sensores. Cada participante realizó tres vueltas, y posteriormente se calculó el promedio de cada parámetro; velocidad $\mathrm{m} / \mathrm{s}$, longitud del paso y longitud de zancada se registran en $\mathrm{cm}$, cadencia pasos/min, el tiempo de paso s, del ciclo del paso, de balanceo, de postura y de apoyo simple se registran en $\mathrm{s}$.

El sistema se considera confiable, debido a que se reporta una fiabilidad test-retest en los parámetros espacio temporales de la marcha con un CCI $\geq 0,92$ IC 95\% [0,84-0,99], a excepción del ancho de la base de apoyo, CCI $=0,80$ IC 95\% [0,50-0,92] (26) y una validez concurrente comparado con el sistema Clinical Stride Analyser en velocidades preferente: velocidad, la longitud de zancada y la ca- 
dencia $(\mathrm{CCI}=0,99)$, tiempo de apoyo con la pierna derecha $(\mathrm{CCI}=0,52)$, tiempo de apoyo con la pierna izquierda $(\mathrm{CCI}=0,76)$ y el porcentaje del ciclo de la marcha en el doble apoyo para ambas piernas $(\mathrm{CCI}=0,44)(27)$.

Para valorar el equilibrio se utilizó la $\mathrm{Ba}$ tería Corta de Desempeño Físico (28) que comprende tres tests: equilibrio, marcha $y$ levantarse y sentarse de una silla. Para este se consideró el test de equilibrio. El equilibrio de pie consiste en las siguientes posiciones: lado por lado (los pies juntos), semitándem (un pie casi delante del otro), tándem (un pie delante del otro). Los posibles puntajes van 1 a 4 puntos, un puntaje inferior a 3 puntos resulta en equilibrio comprometido y mayor a 3 mejor rendimiento del equilibrio. Para el test de equilibrio se reporta una fiabilidad con un Coeficiente de correlación intraclase $=0,6$ (IC 95\%: 0,35-0,70) (29).

La valoración de la sensibilidad protectora de los miembros inferiores se realizó mediante un monofilamento de Semmes-Weinstein, North Coast de 10 g. Se colocó el monofilamento en 10 puntos de la planta del pie. Se dio una calificación de 0 cuando el AM sintió el monofilamento y una calificación de 1 si no lo sentía. Se sumaron los puntajes, se consideró normal cuando sentían 8 de 10 aplicaciones; disminuida de 1 a 7 , y ausente cuando ninguna aplicación era sentida (30). Para el monofilamento se reporta una fiabilidad comparada con la prueba umbral de percepción de la vibración cuantitativa, considerada como una referencia estándar (Kappa = 0,$74 ; r=0,89$ a 0,93 ) y para la prueba umbral de percepción de la vibración cuantitativa ( $\mathrm{ICC}=0,77$ a 0,94; Kappa $=0,74)$. Con una sensibilidad (36\%), especificidad (92\%), valores predictivos positivos $(80 \%)$ y negativos (61\%) (31).

El peso se obtuvo con la báscula mecánica de piso marca SECA 762, con una capacidad de $150 \mathrm{~kg}$ y la estatura se midió con un esta- diómetro marca SECA 274. Posteriormente se obtuvo el IMC con la fórmula de peso/estatura $^{2}$. Los índices se clasificaron como riesgo de desnutrición por bajo peso $\leq 25$, peso normal entre 25,5 a 29,9 y obesidad $>30,0$ $(32,33)$.

\section{Procedimiento}

Se obtuvieron las aprobaciones del Comité de Investigación y Ética en Investigación de la Facultad de Enfermería de la Universidad Autónoma de Nuevo León. Previo a la recolección de los datos sociodemográficos, se contó con el consentimiento informado de los participantes del estudio. Posteriormente se realizó la prueba de la marcha y del equilibrio y después la exploración de pies y el peso y la talla.

\section{Análisis de los resultados}

Los datos se analizaron mediante Statistical Package for the Social Sciences (SPSS) versión 20.0. Se utilizaron medidas de tendencia central y de dispersión. Para determinar la normalidad de la distribución de los datos se utilizó la prueba de Kolmogorov-Smirnov con corrección de Lilliefors. Se utilizó el coeficiente de correlación de Spearman para determinar la relación entre el IMC y parámetros de la marcha y el equilibrio. En virtud de que la sensibilidad de los pies se categorizó en dos grupos: sensibilidad normal y disminuida, se utilizó la prueba U de Mann-Whitney para establecer diferencia de medianas en los parámetros de la marcha y equilibrio.

\section{RESULTADOS}

Participaron 119 adultos mayores (mujeres $=105$ y hombres $=14$ ). La edad promedio 
fue 69,34 años $(\mathrm{DE}=3,17)$ y la escolaridad de 4,70 años $(\mathrm{DE}=3,08)$. Sólo dos participantes refirieron padecer retinopatía diabética. El promedio del tiempo de diagnóstico de DT2 fue de 11,4 años. Cerca de la mitad de la muestra presentaba sensibilidad de pies disminuida $42,9 \%$. Se describen las características de los participantes por nivel de sensibilidad protectora de los pies (Tabla 1).

Antes de realizar el análisis estadístico de acuerdo al nivel de sensibilidad de los pies (normal, disminuida y ausente) con los parámetros de la marcha y el equilibrio, se observó que solo cuatro participantes calificaron en la categoría de severamente afectado por lo que se agregaron a la categoría de dismi- nuida. Se usó la prueba U de Mann-Whitney con dos grupos, normal y disminuida, para analizar las diferencias de medianas en los parámetros de la marcha y el equilibrio. Se encontraron diferencias en la velocidad, longitud del paso, de la zancada y el equilibrio entre aquellos que tenían sensibilidad disminuida y normal (Tabla 2).

Para la asociación entre el IMC con los parámetros de la marcha y el equilibrio, se encontró que el IMC se relaciona con la cadencia, es decir más pasos por segundo $\left(r_{\mathrm{s}}=0,19\right.$, $\mathrm{p}=\leq 0,05)$, disminución del tiempo del ciclo $\left(r_{s}=-0,18, p=\leq 0,05\right)$, tiempo de balanceo $\left(r_{s}=\right.$ $-0,43, p=\leq 0,01)$ y disminución del tiempo de apoyo simple $\left(\mathrm{r}_{\mathrm{s}}=-0,44, \mathrm{p}=\leq 0,01\right)$.

Tabla 1. Características de los participantes por nivel de sensibilidad.

\begin{tabular}{|c|c|c|c|c|c|c|}
\hline \multirow{3}{*}{ Variables } & \multicolumn{6}{|c|}{ Nivel de sensibilidad } \\
\hline & \multicolumn{2}{|c|}{ Normal $n=64$} & \multicolumn{2}{|c|}{ Disminuida $\mathrm{n}=51$} & \multicolumn{2}{|c|}{ Ausente $\mathrm{n}=4$} \\
\hline & $f$ & $\%$ & $f$ & $\%$ & $f$ & $\%$ \\
\hline \multicolumn{7}{|l|}{ Sexo } \\
\hline Masculino $n=14$ & 5 & 7,8 & 8 & 15,7 & 1 & 25 \\
\hline Femenino $\mathrm{n}=105$ & 59 & 92,2 & 43 & 84,3 & 3 & 75 \\
\hline \multicolumn{7}{|l|}{ Estado civil } \\
\hline Con pareja $n=68$ & 23 & 35,9 & 27 & 52,9 & 1 & 25 \\
\hline Sin pareja $\mathrm{n}=51$ & 41 & 64,1 & 24 & 47,1 & 3 & 75 \\
\hline \multicolumn{7}{|l|}{ Nivel de equilibrio } \\
\hline Comprometido $n=67$ & 31 & 48,4 & 34 & 66,7 & 2 & 50 \\
\hline No comprometido $\mathrm{n}=52$ & 33 & 51,6 & 17 & 33,3 & 2 & 50 \\
\hline \multicolumn{7}{|l|}{ Clasificación del IMC } \\
\hline Bajo peso $n=19$ & 7 & 10,9 & 12 & 23,5 & 0 & 0 \\
\hline Peso normal $n=40$ & 24 & 37,5 & 14 & 27,5 & 2 & 50 \\
\hline Obesidad $n=60$ & 33 & 51,6 & 25 & 49 & 2 & 50 \\
\hline
\end{tabular}


Tabla 2. Prueba de U de Mann-Whitney para los parámetros de la marcha y el equilibrio por sensibilidad disminuida y normal.

\begin{tabular}{lcccc}
\hline & $\begin{array}{c}\text { Sensibilidad } \\
\text { normal } \\
\mathrm{n}=64 \\
\text { Mediana }\end{array}$ & $\begin{array}{c}\text { Sensibilidad } \\
\text { disminuida } \\
\mathrm{n}=55 \\
\text { Mediana }\end{array}$ & $\begin{array}{c}\text { U de Mann- } \\
\text { Whitney }\end{array}$ & Valor p \\
\hline Velocidad (cm/s) & 93,71 & 87,03 & 1361 & $\mathbf{0 , 0 3 3}$ \\
Longitud del paso (cm) & 53,25 & 49,1 & 1347 & $\mathbf{0 , 0 2 8}$ \\
Longitud de zancada (cm) & 107,46 & 88,05 & 1321 & $\mathbf{0 , 0 1 9}$ \\
Cadencia & 106,43 & 104,16 & 1520 & 0,201 \\
Tiempo de paso $(\mathrm{s})$ & 0,56 & 0,58 & 1512,5 & 0,187 \\
Tiempo del ciclo $(\mathrm{s})$ & 1,12 & 1,14 & 1523 & 0,207 \\
Tiempo de balanceo $(\mathrm{s})$ & 0,38 & 0,38 & 1717 & 0,819 \\
Tiempo de postura $(\mathrm{s})$ & 0,74 & 0,76 & 1498,5 & 0,163 \\
Tiempo de apoyo simple $(\mathrm{s})$ & 0,38 & 0,38 & 1759,5 & 0,998 \\
Equilibrio & 4,00 & 3,00 & 1241,5 & $\mathbf{0 , 0 0 3}$ \\
\hline
\end{tabular}

\section{DISCUSIÓN Y CONCLUSIÓN}

Este estudio contempló un rango de edad de 65 a 75 años de edad, debido a que mayor edad implica una serie de cambios morfológicos y fisiológicos en todos los tejidos, principalmente en el sistema muscular, en donde el adulto mayor pierde fuerza muscular y además alteran el patrón de la marcha y el equilibrio $(11,34)$. Sin embargo, la media de edad de los participantes fue similar a otros estudios $(17,19,35)$. Otras investigaciones incluyeron participantes desde los 35 ó 40 años de edad $(36,37)$.

La razón de elegir participantes con DT2 es que se considera no curable: con el paso del tiempo los pacientes suelen presentar severas complicaciones si no se tratan adecuada y oportunamente, además estas secuelas generan discapacidades en los adultos mayores y por ende pierden autonomía y grados de independencia.

La media de tiempo de diagnóstico para la DT2 fue de 136,10 meses, que equivale aproximadamente a 11,4 años similar a lo reporta- do por Allet (13) y menor a Palma et al. (16), sin embargo en el estudio de Palma et al. (16) participaron adultos mayores diagnosticados con diabetes tipo 1 y tipo 2. Escasos estudios no reportan el tiempo de duración de la diabetes, sin embargo, es necesario conocer la duración de la enfermedad, debido que conforme aumenta el tiempo de diagnóstico de la enfermedad hay mayor riesgo de presentar complicaciones (38).

Los adultos mayores con DT2 a medida que disminuye la sensibilidad periférica de los pies, mayor es la longitud de la zancada y menor equilibrio, hallazgos consistentes con lo reportado por otros estudios $(12,15,16)$. En las personas con diabetes que desarrollan neuropatía están implicados componentes isquémicos y metabólicos, que inducen cambios que aumentan la resistencia vascular endotelial reduciendo el flujo sanguíneo a los nervios (39), incluyendo fibras del dolor, neuronas motoras, que afecta la biomecánica de las extremidades inferiores, lo que a su vez lleva a alteraciones de la marcha y equilibrio con el aumento del riesgo de caídas (13).

El IMC se asoció a mayor cadencia, dis- 
minución del tiempo de paso, tiempo del ciclo, tiempo de balanceo y disminución del tiempo de apoyo simple. Estos resultados son similares a los reportados por autores (19, 40) que realizaron el estudio en población similar, lo que confirma que a medida que aumenta el IMC en los adultos mayores con DT2, mayor es la alteración de los patrones de la marcha y el equilibrio.

Lo anterior se explica dado que al llegar al envejecimiento el AM pierde masa magra, que si no es balanceado con una disminución de la ingesta, lleva a la ganancia de peso lenta e irremediablemente (41), y este aumento del peso corporal contribuye al aumento de la carga mecánica y dinámica en los patrones del aparato locomotor, principalmente en los movimientos de la rodilla $(22,40)$.

Los parámetros de la marcha y el equilibrio se asocian con la sensibilidad de los pies y el IMC en adultos mayores con presencia de DT2. Por lo tanto, las personas con estas características tienen mayor riesgo de caídas y con ello fracturas y hospitalizaciones.

En los programas de prevención de caída es de gran importancia la correcta identificación de los pacientes en riesgo, por lo que es necesario realizar la oportuna identificación del riesgo de caída para prevenir daños biopsicosociales, así mismo de realizar medidas de protección a los adultos mayores con alteraciones de la marcha y el equilibrio.

Es por ello que esta información es de gran utilidad para que el personal de enfermería, en el primer nivel de atención, valore la pérdida de sensibilidad de los pies, con una herramienta de fácil manejo como los monofilamentos, así mismo llevar un buen control de sobrepeso / obesidad, y de tal manera prolongar la afectación de la marcha y el equilibrio en el adulto mayor y disminuir el riesgo de las caídas. Por otra parte, los resultados sugieren que los parámetros de la marcha y el equilibrio pueden ser considerados en modelos explicativos o predictivos, en riesgo de caídas en adultos mayores con DT2.

La principal limitación de este estudio fue el tipo de diseño; en virtud de que el estudio fue de tipo transversal las variables se midieron en un solo momento del tiempo, lo que limita su evaluación de manera precisa. No se puede generalizar porque se utilizó un muestreo no probabilístico.

Se sugiere para posteriores estudios, por una parte, incluir diseños de cohorte en adultos con DT2, por otra, controlar la población por sexo, debido a que la obesidad prevalece en el sexo femenino, este fenómeno se asocia con la maternidad (42), es decir que al término del embarazo la mayoría de las mujeres no recuperan el peso corporal anterior. De igual modo, se sugiere diagnosticar las complicaciones de la DT2, como son la neuropatía y retinopatía, con instrumentos estandarizados, considerando que estas complicaciones pueden influir en las alteraciones de la marcha y el equilibrio.

\section{REFERENCIAS}

1. United Nations (Department of Economic and Social Affairs, Population Division). World Population Ageing 2013 [Internet]. New York: United Nations; 2013 [citado 10 octubre 2014]. 95 p. Disponible en: http://www.un.org/en/development/desa/population/publications/ pdf/ageing/WorldPopulationAgeingReport2013.pdf

2. Organización Mundial de la Salud (OMS). 10 datos sobre el envejecimiento y la salud [Internet]. Ginebra: OMS; 2015 [citado 10 octubre 2014]. Disponible en: http://www.who.int/features/factfiles/ ageing/es/

3. Consejo Nacional de Población (CONA$\mathrm{PO}$ ). La situación demográfica de México [Internet]. México, D. F: CONAPO; 2013[citado 10 octubre 2014]. 190 p. Disponible en: http://www.conapo.gob. $\mathrm{mx} /$ work/models/CONAPO/Resource/2468/2/images/SDM_2013.pdf 
4. Manrique-Espinoza B, Salinas-Rodríguez A, Moreno-Tamayo KM, Acosta-Castillo I, Sosa-Ortiz AL, Gutiérrez-Robledo LM, et al. Condiciones de salud y estado funcional de los adultos mayores en México. Salud Publica Mex. 2013; 55(2): S323-S31.

5. Frontera WR, Suh D, Krivickas LS, Hughes VA, Goldstein R, Roubenoff R. Skeletal muscle fiber quality in older men and women. Am J Physiol Cell Physiol. 2000; 279(3): C611-8.

6. Yu M, Piao YJ, Eun HI, Kim DW, Ryu MH, Kim NG. Development of abnormal gait detection and vibratory stimulation system on lower limbs to improve gait stability. J Med Syst. 2010; 34(5): 787-97.

7. Demirbüken İ, İlçin N, Gürpinar B, Algun C. The Effect of Type 2 Diabetes Mellitus on The Motor Behaviour of Elderly Individuals During Sit to Stand Activity. MÜSBED. 2012; 2(2): 72-7.

8. Salzman B. Gait and balance disorders in older adults. Am Fam Physician; 2010; 82(1): 61-8.

9. Inman VT, Ralston HJ, Todd F. Human walking. Baltimore, London:Williams \& Wilkins; 1981.

10. Shumway-Cook A, Woollacott MH. Motor control: translating research into clinical practice. 3ra ed. Philapelphia: Lippincott Williams \& Wilkins; 2007.612 p.

11. Cerda AL. Manejo del trastorno de marcha del adulto mayor. Revista Médica Clínica Las Condes. 2014;25(2) :265-75.

12. LER: Lower Extremity Review [Internet]. NY: LER (Khan T, Guberman R); 20092017. Gait alterations associated with diabetic neuropathy; 2012 Ago [citado 5 nov 2014]. Disponible: http://lermagazine.com/article/gait-alterations-associated-with-diabetic-neuropathy.

13. Allet L. Gait and balance characteristics in patients with diabetes type 2: evaluation and treatment efficacy [dissertation]. Holanda: Universitaire Pers Maastricht; 2009.
14. Cesari M, Kritchevsky SB, Penninx BW, Nicklas BJ, Simonsick EM, Newman AB, et al. Prognostic value of usual gait speed in well-functioning older people-results from the Health, Aging and Body Composition Study. J Am Geriatr Soc. 2005; 53(10): 1675-80.

15. Lim KB, Kim DJ, Noh JH, Yoo J, Moon JW. Comparison of balance ability between patients with type 2 diabetes and with and without peripheral neuropathy. PM R. 2014; 6(3): 209-214.

16. Palma FH, Antigual DU, Martínez SF, Monrroy MA, Gajardo RE. Static balance in patients presenting diabetes mellitus type 2 with and without diabetic polyneuropathy. Arq Bras Endocrinol Metabol. 2013; 57(9): 722-6.

17. Wuehr M, Schniepp R, Schlick C, Huth S, Pradhan C, Dieterich M, et al. Sensory loss and walking speed related factors for gait alterations in patients with peripheral neuropathy. Gait Posture. 2014; 39(3): 852-8.

18. Wearing SC, Hennig EM, Byrne NM, Steele JR, Hills AP. The biomechanics of restricted movement in adult obesity. Obes Rev 2006; 7(1): 13-24.

19. Ko S, Stenholm S, Ferrucci L. Characteristic gait patterns in older adults with obesity-results from the Baltimore Longitudinal Study of Aging. J Biomech. 2010; 43(6): 1104-10.

20. Spyropoulos P, Pisciotta JC, Pavlou KN, Cairns MA, Simon SR. Biomechanical gait analysis in obese men. Arch Phys Med Rehabil. 1991; 72(13): 1065-70.

21. Friedmann JM, Elasy T, Jensen GL. The relationship between body mass index and self-reported functional limitation among older adults: a gender difference. J Am Geriatr Soc. 2001; 49(4): 398-403.

22. Maffiuletti NA, Agosti F, Proietti M, Riva D, Resnik M, Lafortuna CL, et al. Postural instability of extremely obese individuals improves after a body weight reduction program entailing specific balance tra- 
ining. J Endocrinol Invest. 2005; 28(1): 2-7.

23. Lai PP, Leung AK, Li AN, Zhang M. Three-dimensional gait analysis of obese adults. Clin Biomech. 2008; 23 Suppl 1:S2-S6.

24. NANDA Internacional. Diagnósticos enfermeros: definiciones y clasificación 2012-2014. Barcelona: Elsevier; 2012. 616 p.

25. Cohen J. Statistical power analysis for the behavioural sciences. Hillside, NJ: Lawrence Erlbaum Associates; 1988. 400 $\mathrm{p}$.

26. Van Unden CJ, Besser MP. Test-retest reliability of temporal and spatial gait characteristics measured with an instrumented walkway system (GAITRite). BMC Musculoskelet Disord [Internet]. 2004 [citado 5 nov 2014]; 5:13. Disponible en: http:// bmcmusculoskeletdisord.biomedcentral. com/articles/10.1186/1471-2474-5-13

27. Bilney B, Morris M, Webster K. Concurrent related validity of the GAITRite walkway system for quantification of the spatial and temporal parameters of gait. Gait Posture. 2003; 17(1): 68-74.

28. Guralnik JM, Simonsick EM, Ferrucci L, Glynn RJ, Berkman LF, Blazer DG et al. A short physical performance battery assessing lower extremity function: association with self-reported disability and prediction of mortality and nursing home admission. J Gerontol. 1994; 49(2): M85-94.

29. Cabrero-García J, Muñoz-Mendoza CL, Cabañero-Martínez MJ, González-Llopís L, Ramos-Pichardo JD, Reig-Ferrer A. Valores de referencia de la Short Physical Performance Battery para pacientes de 70 y más años en atención primaria de salud. Aten Primaria. 2012; 44(9): 540-8.

30. Feldman EL, Stevens MJ, Thomas PK, Brown MB, Canal N, Greene DA. Practical two-step quantitative clinical and electrophysiological assessment for the diagnosis and staging of diabetic neuro- pathy. Diabetes Care. 1994; 17(11), 12811289.

31. Shaffer S, Harrison A, Brown K, Brennan K. Reliability and validity of semmes-weinstein monofilament testing in older community-dwelling adults. J Geriatr Phys Ther. 2005; 28(3): 112-3.

32. Kvamme JM, Olsen JA, Florholmen J, Jacobsen BK. Risk of malnutrition and health-related quality of life in community-living elderly men and women: the Tromso study. Qual Life Res. 2011; 20(4): 575-82.

33. World Health Organization Working Group. Use and interpretation of anthropometric indicators of nutritional status. Bull World Health Organ. 1986; 64(6): 929-941.

34. Salech MF, Jara LR, Michea AL. Cambios fisiológicos asociados al envejecimiento. Rev. Med. Clin. Condes. 2012; 23(1): 1929.

35. Manor B, Li L. Characteristics of functional gait among people with and without peripheral neuropathy. Gait Posture. 2009; 30(2): 253-6.

36. Mueller MJ, Minor SD, Sahrmann SA, Schaaf JA, Strube MJ. Differences in the Gait Characteristics of Patients with Diabetes and Peripheral Neuropathy Compared with Age-Matched Controls. Phys Ther. 1994; 74(4): 299-308.

37. Petrofsky J, Lee S, Bweir S. Gait characteristics in people with type 2 diabetes mellitus. Eur J Appl Physiol. 2005; 93(5-6): 640-7.

38. International Diabetes Federation. IDF Global Guideline for Managing Older People with Type 2 Diabetes [Internet]. Belgium: IDF; 2013 [citado 5 nov 2014]. 94 p. Available from: http://www.idf.org/ sites/default/files/IDF-Guideline-for-older-people-T2D.pdf

39. National Institutes of Health. National Institute of Diabetes and Digestive and Kidney Diseases. Neuropatías diabéticas: el daño de los nervios en personas 
con diabetes [Internet]. 2012 [citado 05 noviembre 2014]. Disponible en: https://www.niddk.nih.gov/health-information/informacion-de-la-salud/ diabetes/prevenir-problemas-diabetes/ neuropatas-diabticas-dano-nervios-personas-diabetes.

40. Harding GT, Hubley-Kozey CL, Dunbar MJ, Stanish WD, Astephen Wilson JL. Body mass index affects knee joint mechanics during gait differently with and without moderate knee osteoarthritis. Osteoarthritis Cartilage. 2012; 20(11): 1234-42.
41. Rodríguez Scull LE. Obesidad: fisiología, etiopatogenia y fisiopatología. Rev Cubana Endocrinol [Internet]. 2003 [citado 5 nov 2014]; 14(2). Disponible en: http:// scielo.sld.cu/scielo.php?script=sci_arttext\&pid=S1561-29532003000200006

42. Olson CM, Strawderman MS, Dennison BA. Maternal Weight Gain During Pregnancy and Child Weight at Age 3 Years. Matern Child Health J. 2009; 13(6): 839846. 